\title{
Recent trends in the BSE epidemic
}

Sir - The confirmation of 21 cases of new variant Creutzfeldt-Jakob disease (vCJD) in humans by 31 August 1997 and the continuing ban on the export of beef and cattle from the United Kingdom have stimulated a continuing interest in the past, present and future course of the bovine spongiform encephalopathy (BSE) epidemic in the United Kingdom. We report analyses of the latest available BSE case data (for which we are grateful to J. Wilesmith and colleagues at the Central Veterinary Laboratory) and discuss their implications for future trends in the light of recent culling programmes.

By 21 August 1997, 168,578 cases of BSE had been confirmed in Great Britain (GB). Analyses of the maternal cohort study ${ }^{1-5}$ and the GB case database ${ }^{6}$ have revealed an enhanced risk of BSE in calves born to infected dams, with the cohort study suggesting a maternal transmission rate of approximately 10 per cent over the last 5 months of the maternal incubation period. Analysis of dam-calf pairs in the case database revealed an enhanced risk in calves born up to 2 years before the onset of clinical signs of BSE in the dam with the greatest risk in those born after onset ${ }^{6}$.

The observed number of cases onset in $1996(7,417)$ is in close agreement with the numbers predicted in recent analyses of the epidemic $(7,386$ with a 95 per cent prediction interval (PI), 6,541-8,856) (Figure 1) (ref. 7). So far, 1,659 BSE cases have been confirmed with clinical onset in 1997. Extrapolating from the cases already seen in 1997 and adjusting for the delay between reported clinical onset to confirmation by pathology, 3,592 cases are estimated for 1997 _ consistent with model predictions (95 per cent PI, 3,006-7,664) (ref. 7).

Two control programmes based on culling of GB cattle have been implemented over the past 18 months, namely, culling of animals over 30 months of age (OTMS) introduced in May 1996 and a selective cull

\section{Rules needed on}

\section{authorship}

Sir - You report (Nature 389, 105; 1997) that Dr Friedhelm Herrmann's lawyers argue that as senior author he would not have had any responsibility for possibly fabricated data and, moreover, that a full professor would not have a motive to commit fraud. Both statements are ridiculous.

Any scientist has to publish, or reputation and grant-money will be seriously endangered. The only way to publish for scientists no longer doing bench-work

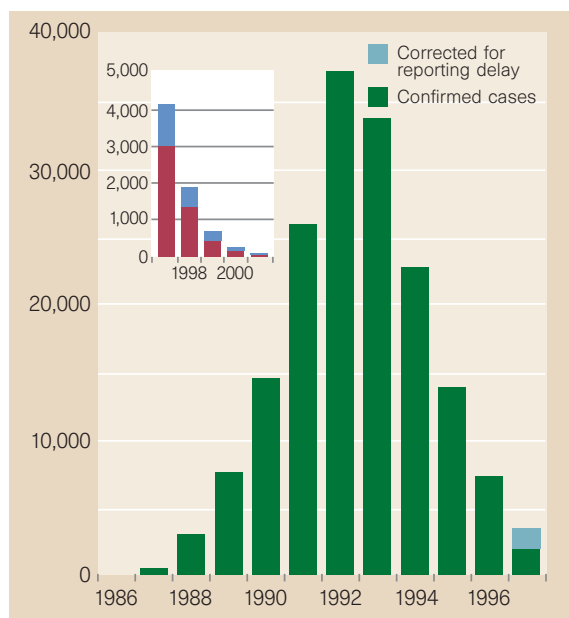

Figure 1 Number of BSE cases by year of clinical onset. The number of cases onset in 1997 was adjusted for confirmation delay by month for January to June and the monthly incidence for July to December was set equal to the adjusted number for June. An adjustment by month to the number of cases onset in 1996 increased the number from 7,417 to 7,441. Inset: Predictions of cases by year for 1997 to 2001 with no selective cull (blue) and assuming the Ministry of Agriculture selective cull was fully implemented by 1 January 1996 (red) (ref. 7).

initiated in March 1997, targeting herds with a history of BSE in line with the Florence agreement made with the European Commission in June 1996. Under the OTMS, 1,403,619 cattle had been slaughtered by 17 August 1997, but this number is only approximately 10 per cent greater than the expected number of deaths that would have taken place before the introduction of OTMS (based on the GB cattle survival distribution estimated using pre-1996 data $\left.{ }^{7,8}\right)$. By 22 August 1997, 19,271 cattle had been slaughtered under the selective cull agreed with the European Commission. Estimating the impact of both schemes is made difficult by the absence of data on the ages of animals slaughtered under OTMS and limited

themselves is to guide research and be senior authors. A senior author must be responsible for the scientific soundness and honesty of a paper, or he or she should not be in this distinguished place. They get reputation from it after all, and the quality of a paper is often inherently inferred from the name and status of the senior author.

The minimum requirement for senior authorship should be that the paper has been read, understood and worked on by intelligently and critically discussing it with the people who did the actual bench-work. In a perfect world, the senior author will also have provided ideas and mental input information on the animals targeted in the selective cull. However, Figure 1 provides approximate upper and lower bounds for future years.

A recent suspected case of $\mathrm{vCJD}$ in an individual who appears not to have consumed meat and meat products since 1985-86 has triggered further interest in the early stages of the BSE epidemic. Although the first case of BSE in cattle was diagnosed in 1986 (ref. 9), we estimate that up to 54,000 infected animals were slaughtered for human consumption before clinical onset of BSE between 1980 and 1985, depending on the level of under-reporting assumed ${ }^{10}$. Overall, before the introduction of the specified bovine offal ban in 1989, 480,000 infected animals were slaughtered for consumption ${ }^{10}$. Most of these animals were in the early stages of the incubation period. Since the incubation period distribution of vCJD is unknown, it is not possible at present to produce meaningful predictions of future trends in vCJD cases. This uncertainty will continue for the foreseeable future.

\section{A. Donnelly}

\section{A. C. Ghani}

N.M. Ferguson

R. M. Anderson

Wellcome Trust Centre for the

Epidemiology of Infectious Disease,

Department of Zoology,

University of Oxford,

South Parks Road,

Oxford OX1 3PS,UK

e-mail:christl.donnelly@zoo.ox.ac.uk

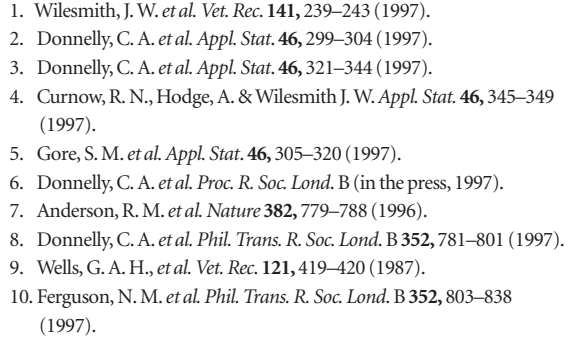

through the course of the experiments and have seen the problems and difficulties of the study. There are heads of laboratories who provide just that, and are therefore rightly named the senior authors. I do not think that - as is sometimes the case - being head of a department or institute, providing laboratory space, or simply giving permission to use costly equipment as such justifies senior authorship. Unfortunately, there are no real rules, but it is time that there were.

C. Esser

Medical Institute of Environmental Hygiene, Aufdem Hennekamp 50, 40225 Düsseldorf, Germany 